\title{
High precision measurements of neutrino fluxes with ENUBET
}

\section{A. Longhin*,}

INFN Sezione di Padova, via Marzolo, 8 - Padova, Italy.

E-mail: andrea. longhin@pd.infn.it

G. Ballerini ${ }^{a, b}$, A. Berra ${ }^{a, b}$ R. Boanta ${ }^{b, h}$ M. Bonesini ${ }^{b}$, C. Brizzolari ${ }^{a, b}$, G. Brunetti ${ }^{j}$,

M. Calviani ${ }^{m}$, S. Carturan ${ }^{k, t}$, M.G. Catanesi $^{l}$, S. Cecchini ${ }^{c}$, F. Cindolo $^{c}$,

A. Coffani ${ }^{b, h}$, G. Collazuol ${ }^{k, j}$, E. Conti ${ }^{j}$, F. Dal Corso ${ }^{j}$, G. De Rosa ${ }^{p, q}$, A. Gola ${ }^{o}$, R.A. Intonti ${ }^{l}$, C. Jollet ${ }^{d}$, Y. Kudenko ${ }^{r}$, M. Laveder ${ }^{k}, j$, P.F. Loverre ${ }^{n, f}$, L. Ludovici ${ }^{f}$, L. Magaletti ${ }^{l}$, G. Mandrioli ${ }^{c}$, A. Margotti ${ }^{c}$, V. Mascagna ${ }^{a, b}$, N. Mauri ${ }^{c}$,

A. Meregaglia ${ }^{s}$, M. Mezzetto ${ }^{j}$, M. Nessi ${ }^{m}$, A. Paoloni ${ }^{e}$, M. Pari ${ }^{k, j}$, G. Paternoster ${ }^{o}$, L. Patrizii ${ }^{c}$, C. Piemonte ${ }^{o}$, M. Pozzato ${ }^{c}$, M. Prest ${ }^{a, b}$, F. Pupilli ${ }^{j}$, E. Radicioni ${ }^{l}$, C. Riccio ${ }^{p, q}$, A.C. Ruggeri ${ }^{p}$, M. Soldani ${ }^{a, b}$ G. Sirri $^{c}$, M. Tenti ${ }^{c, g}$, F. Terranova ${ }^{h, b}$, E. Vallazza ${ }^{i}, \mathbf{M}$. Vesco ${ }^{k, t}$, L. Votano ${ }^{e}$, E. Wildner ${ }^{m}$

${ }^{a}$ DiSAT, Università degli Studi dell'Insubria, via Valeggio 11, Como, Italy

${ }^{b}$ INFN, Sezione di Milano-Bicocca, piazza della Scienza 3, Milano, Italy

${ }^{c}$ INFN, Sezione di Bologna, viale Berti-Pichat 6/2, Bologna, Italy

${ }^{d}$ IPHC, Université de Strasbourg, CNRS/IN2P3, Strasbourg, France

e INFN, Laboratori Nazionali di Frascati, via Fermi 40, Frascati (Rome), Italy

${ }^{f}$ INFN, Sezione di Roma 1, piazzale A. Moro 2, Rome, Italy

${ }^{g}$ Phys. Dep. Università di Bologna, viale Berti-Pichat 6/2, Bologna, Italy

${ }^{h}$ Phys. Dep. Università di Milano-Bicocca, piazza della Scienza 3, Milano, Italy

${ }^{i}$ INFN Sezione di Trieste, via Valerio, 2 - Trieste, Italy

j INFN Sezione di Padova, via Marzolo, 8 - Padova, Italy

${ }^{k}$ Phys. Dep. Università di Padova, via Marzolo, 8 - Padova, Italy

${ }^{l}$ INFN Sezione di Bari, via Amendola, 173 - Bari, Italy

${ }^{m}$ CERN, Geneva, Switzerland

${ }^{n}$ Phys. Dep. Università La Sapienza, piazzale A. Moro 2, Rome, Italy

${ }^{o}$ Fondazione Bruno Kessler (FBK) and INFN TIFPA, Trento, Italy

${ }^{p}$ INFN, Sezione di Napoli, via Cinthia, 80126, Napoli, Italy

${ }^{q}$ Phys. Dep. Università degli Studi di Napoli Federico II, via Cinthia, 80126, Napoli

${ }^{r}$ Institute of Nuclear Research of the Russian Academy of Science, Moscow, Russia

${ }^{s}$ CENBG, Université de Bordeaux, CNRS/IN2P3, 33175 Gradignan, France

${ }^{t}$ INFN Laboratori Nazionali di Legnaro, Viale dell'Università, 2 - 35020 Legnaro (PD), Italy. 
Neutrino fluxes are currently affected by large normalization uncertainties (5-10\%). Neutrino physics will require measurements of absolute neutrino cross sections at the $\mathrm{GeV}$ scale with exquisite (1\%) precision in the near future. For this reason a reduction of the present uncertainties by one order of magnitude would be highly beneficial. This goal might be achieved by producing a sign and momentum selected narrow band beam and monitoring the production of $e^{+}$in the decay tunnel from the decays of charged kaons ( $K_{e 3}$ channel). This technique, which requires a special instrumented beamline, would allow a $1 \%$ level measurement of the cross-sections of the neutrino species $\left(v_{e}\right.$ and $\left.\bar{v}_{e}\right)$ which are the final states involved in the searches for CP violation with muon neutrino beams at long-baseline. The ENUBET Horizon-2020 ERC Consolidator Grant, approved by the European Research Council in 2015, is the framework within which such a non-conventional beamline will be developed. We present a progress report of the project (20162021) after about one year of work, the experimental results on ultra-compact calorimeters suited for instrumenting the decay tunnel and the $R \& D$ in the design of the hadronic beamline.

XVII International Workshop on Neutrino Telescopes

13 - 17 March, 2017

Venice,Italy

${ }^{*}$ Speaker. 


\section{Monitored neutrino beams from Kaon tagging}

The prediction of the neutrino flux in conventional beams is a challenging task relying heavily on a detailed simulation of the beamline elements and on hadronic physics. Systematic uncertainties receive important contributions from the limited knowledge of the kinematics of pions and kaons emerging from proton-target interactions, their reinteractions in the passive elements, the need for describing accurately their trajectories and decays.

Data driven constraints to the simulation have been employed in the past using beam monitoring devices and ancillary measurements: proton intensity, horn currents, muon rates after the beam dump, beam-target alignment etc. Dedicated hadro-production experiments provide the particle yields from the target. Nevertheless flux prediction preserves a critical link with ab-initio simulations leading to large systematics $(\mathscr{O}(5-10 \%))$. Flux uncertainties represent the common limitation for a precision measurement of all neutrino cross section measurements. In particular the $v_{e}$ and $\bar{v}_{e}$ cross sections are poorly known in spite of their relevance for the next generation of long-baseline experiments.

A breakthrough in the field could be achieved by monitoring the production of $e^{+(-)}$from $K_{e 3}$ decays $\left(K^{+(-)} \rightarrow \pi^{0} e^{+(-)} v_{e}\left(\bar{v}_{e}\right)\right)$ directly in the decay tunnel employing a sign and momentumselected narrow band beam [1].

The rate of produced positrons is a direct observable to constrain the flux of $v_{e}$ since these particles are connected simply through the kinematics of the decay and the acceptances of the respective detectors. Due to the large mass of the Kaon, positrons from $K_{e 3}$ decays are produced at an average angle $(\sim 100 \mathrm{mrad})$ much larger than the muon emission angle from the $\pi^{+} \rightarrow \mu^{+} v_{\mu}$ decay. If then the decay tunnel length is kept sufficiently short $(\sim 50 \mathrm{~m})$ and the momentum of secondary hadrons is of a few $\mathrm{GeV}, K_{e 3}$ are left being the only source of $v_{e}$ in the beamline.

\section{The ENUBET ERC project}

The ENUBET project (Enhanced NeUtrino BEams from kaon Tagging) [2] is aimed at demonstrating in a conclusive manner the technical feasibility and physics performance of monitored neutrino beams. This will be achieved by 1) designing and constructing a detector capable of performing positron identification in the harsh environment of a $v$ beam decay tunnel and by 2) studying the precise layout of the $K / \pi$ focusing and transport system with suitable proton extraction schemes. The Project is funded by the European Research Council (ERC Consolidator Grant, PI A. Longhin, host institution INFN) from June 2016 to May 2021.

ENUBET might enable a new generation of neutrino cross section experiments, it could be exploited for sterile- $v$ experiments if present anomalies would be confirmed. In addition, ENUBET aims at being an important step towards a "time-tagged $v$ beam" [2] where $v_{e}$ interactions could be time correlated with an $e^{+}$in the decay tunnel. This direction has been recently boosted since ENUBET has been awarded additional funds from the Italian ministry for education and science (MIUR) to develop a timing system below 1 ns (NUTECH, NeUtrino Time-tagged bEams with CHerenkov detectors). ENUBET is also framed in the CERN Neutrino Platform under the NP3Plafond R\&D programme since December 2016 [2]. 


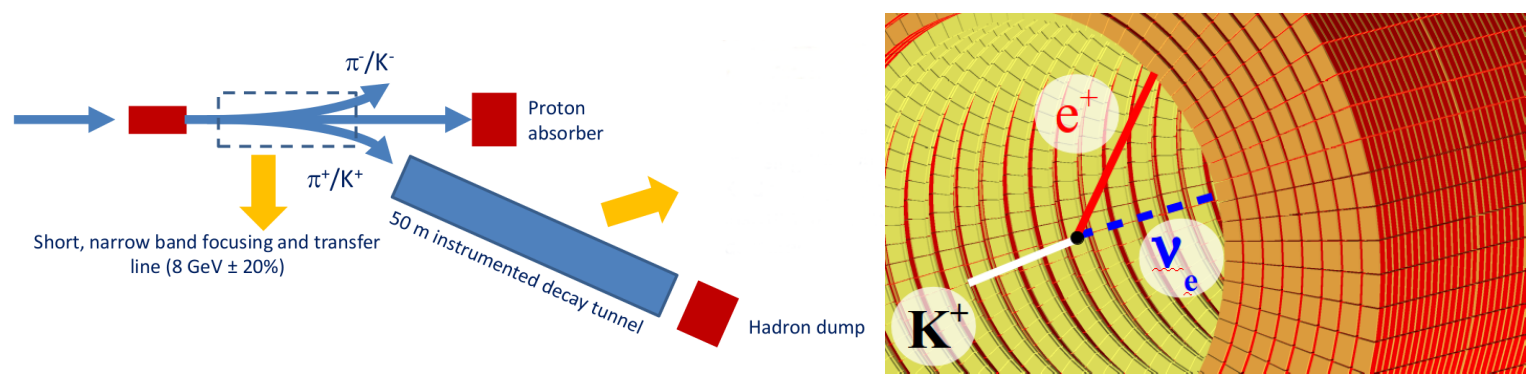

Figure 1: (Left): Layout of the beamline. (Right): A section of the positron tagger. The Ultra Compact Modules (UCMs) are shown in red and the photon veto detector in yellow.

\section{Layout of the ENUBET monitored beam}

In the baseline design the positron tagger is shaped as a hollow cylinder surrounding a fraction of the decay tunnel. It consists of a calorimetric section for $\mathrm{e}^{+}$tagging and $\pi^{ \pm}$rejection (in red in Fig. 1 left) and a system of light tracking devices in the innermost region to reject showers from $\gamma$ conversions (photon veto, yellow).

The secondary beam is designed to have an average momentum of $8.5 \mathrm{GeV}$ and a $\pm 20 \%$ momentum bite. The energy range of the hadronic beam is chosen to allow for a small fraction of $K$ decaying in the transfer line and the possibility to tag positrons with purely calorimetric techniques. It also allows the neutrino energy spectrum to lie in the region of interest for future long baseline experiments. These energies are also high enough to keep the contamination of $v_{e}$ from $\mu$ decays to a very small level (below $2 \%$ ). The emittance at the entrance of the decay tunnel (few mrad over $10 \times 10 \mathrm{~cm}^{2}$ ) is driven by the necessity to prevent undecayed secondaries or $\mu$ from $\pi$ decay from hitting the tagger causing unmanageable pile-up effects. Under this assumption the rates in the target are completely dominated by Kaon decays emitting secondaries at large angles.

\section{Design of the hadronic beamline}

The hadronic beamline has to be designed to focus and transport a collimated beam of signand momentum-selected pions and Kaons with high efficiency, limited losses from decays and low beam-induced backgrounds. The ENUBET collaboration (Work Package 1) is developing the focusing system both assuming a conventional pulsed horn and a static system for different options for the incoming proton energy (30,120 and $400 \mathrm{GeV} / \mathrm{c})$. The optimization of the focusing system and the design of the transfer line is on a good track and more realistic layouts confirm or even improve the collection efficiencies assumed in [1]. In particular, the optimization of the line (dipoles and quadrupoles) is performed with TRANSPORT [3]. The implementation and full simulation of particle transport, collimation and re-interaction is carried out with G4Beamline. Assessment of the doses is addressed using FLUKA [4, 5]. A candidate layout for a static-based transfer line simulated with G4Beamline is shown in Fig. 2.

The positron tagger can be safely operated in terms of pile-up, if local particle rates are below $\sim 500 \mathrm{kHz} / \mathrm{cm}^{2}$. This can be achieved with multi-Hz slow extractions with a $\mathscr{O}(\mathrm{ms})$ duration. Extractions significantly longer than $10 \mathrm{~ms}$ are disfavored if focusing of secondaries is achieved by 


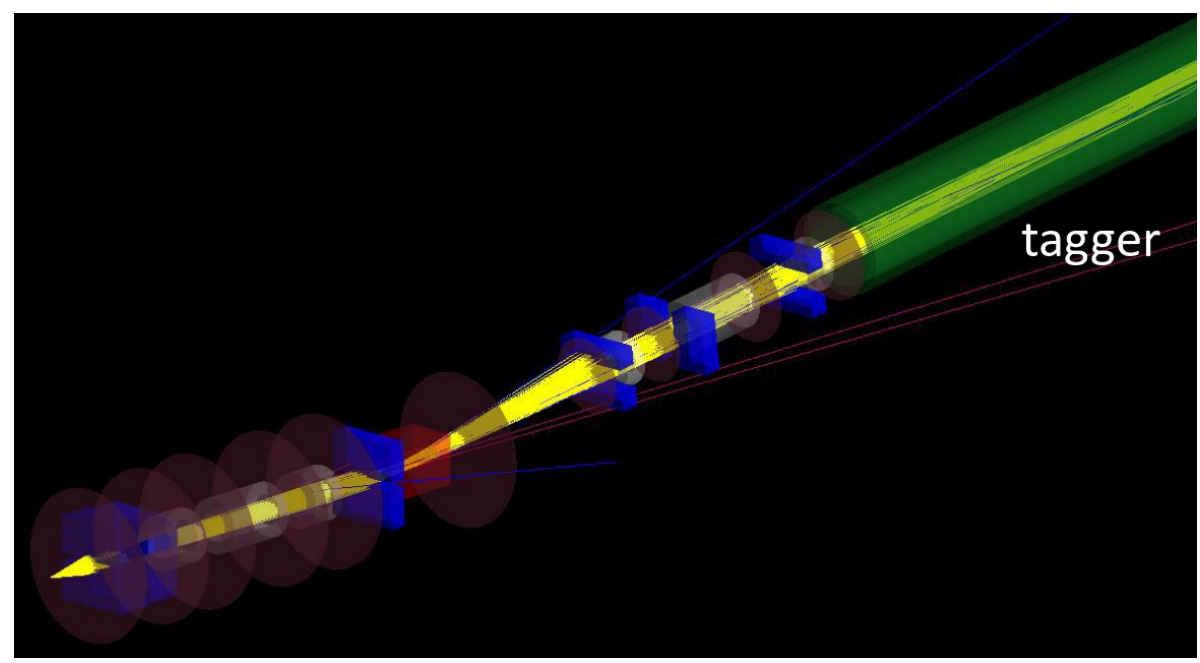

Figure 2: Preliminary layout of the hadronic beamline based on an upstream quadrupole triplet, bending dipole and downstream quadrupole triplet. The drawing and simulation is performed with G4BeamLine. Charged particle trajectories in the selected momentum bite are in yellow, collimators in blue, the dipole in red, quadrupoles in gray and the positron tagger in green. The proton and hadron dumps are not shown for simplicity.

magnetic horns. This constraint could be removed by designing a very efficient focusing system based on DC operated magnets. The static focusing system has been proved to be typically less efficient than the one based on pulsed magnetic horns. On the other hand employing the static system has the advantage of mitigating the pile-up and easing the possibility of a time-tagged configuration; it would remove risks related to mechanical stresses on the horn and the need for less conventional extraction schemes (multi-Hz). These two scenarios are being studied in depth: proton extraction schemes compatible with accelerators at CERN, Fermilab and J-PARC are considered and machines studies at the SPS are foreseen before the next CERN long-shutdown.

\section{Simulation of the positron tagger}

A GEANT4 [6] simulation of the decay tunnel and instrumentation has been completed within Work Package 5 in parallel with the simulation of the transfer line. Assuming the rates of $[1](<500$ $\mathrm{kHz} / \mathrm{cm}^{2}$ ), a reconstruction algorithm has been developed capable of coping with pile-up effects in realistic conditions. The algorithms for particle identification [7, 8] employ the energy deposit pattern in the calorimeter modules to discriminate electromagnetic from hadronic showers. This allows selecting a sample enriched in positrons from $K_{e 3}$ decays. The timing information from both the shashlik calorimeter UCMs and the photon veto is exploited to cluster modules belonging to the same decay ("event building") and to reject electromagnetic showers generated by the conversions of photons from $\pi^{0}$ decays.

The calorimetric modules are positioned at a $1 \mathrm{~m}$ radius from the axis of the decay tunnel. Positron efficiencies and purities have been estimated on the basis of the rate and particle distribution of [1]. The ENUBET instrumentation provides positron identification with an efficiency of 
$25 \%$ and a signal-to-noise ratio of $\sim 1$. These figures are being updated profiting of the recent inputs from the simulation of the transfer line. These ingredients allow to fully take into account beam related backgrounds and will likely introduce the possibility to operate at reduced particle rates. The assessment of the systematics in the neutrino flux due to the detector response is ongoing. More details on this topic can be found in the report by F. Pupilli at this conference [8].

Ionizing and non-ionizing doses (from neutrons) have been evaluated with FLUKA 2011 [4, 5] at the inner surface of the target where they are more severe. They are shown in Fig. 3 (left) for different choices of the inner radius of the tagger: 40,80 and $100 \mathrm{~cm}$. These integrated doses correspond to $10^{4} v_{e}^{C C}$ events at the neutrino detector and amount to $<0.05 \mathrm{kGy}$ and $1.9 \times 10^{11} \mathrm{n} / \mathrm{cm}^{2}$ ( $1 \mathrm{MeV}$ equivalent) for $1 \mathrm{~m}$ radius. These estimates validate the possibility of using conventional plastic scintillators as an active medium for the calorimeter and photon veto. It can be noticed in Fig. 3 (right) that an additional consistent mitigation (by more than a factor 6) of the irradiation levels could be achieved by placing the photosensors outside of the calorimeter by adopting a non-shashlik solution with radial routing of the scintillation light.
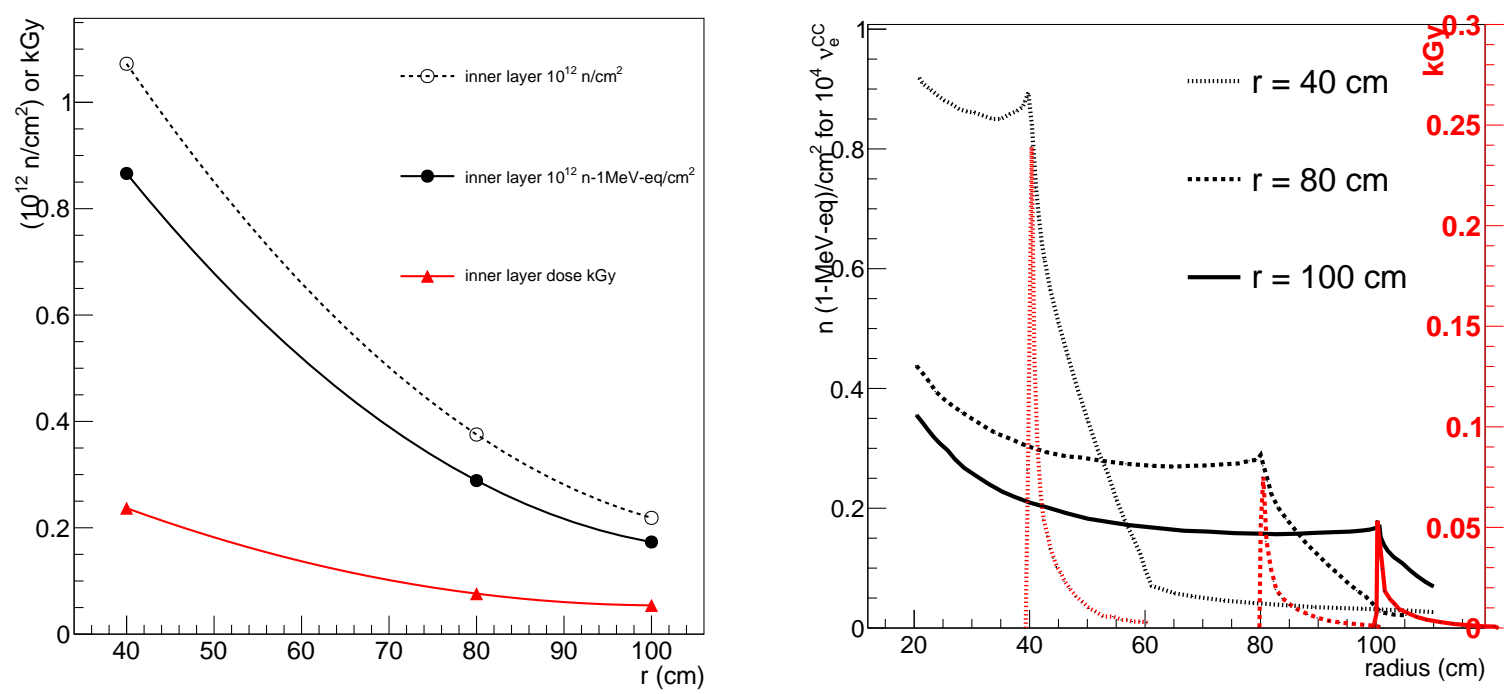

Figure 3: (Left): Ionizing dose (red line, kGy), neutron fluence (black dashed line, in $\mathrm{n} / \mathrm{cm}^{2}$ ) and $1 \mathrm{MeV}$ equivalent neutron fluence (black continuous line) in the innermost modules of the calorimeter as a function of the tagger radius. (Right): Radial dependence of the 1-MeV-eq. neutron dose for three tagger configurations with an inner radius of 40,80 or $100 \mathrm{~cm}$.

The tunnel and detector geometry has been re-optimized with respect to [1] during the first year of the project leading to an improvement in the physics performances. A $500 \mathrm{t}$ neutrino detector with a transverse size of $6 \times 6 \mathrm{~m}^{2}$ (similar to ICARUS at Fermilab or ProtoDune-SP/DP at CERN) located $50 \mathrm{~m}$ downstream of the tagger is capable of recording $10^{4} v_{e}^{C C}$ events $(1 \%$ overall statistical uncertainty on $\sigma\left(v_{e}\right)$ ) with $1.0 \times 10^{20}$ protons on target (pot) at the J-PARC synchrotron $(30 \mathrm{GeV})$. The same result can be obtained at the Fermilab Main Ring $(120 \mathrm{GeV})$ with $2.4 \times 10^{19}$ pot and at the CERN SPS $(400 \mathrm{GeV})$ with $1.1 \times 10^{19}$ pot. These figures correspond to 2-3 months of 
data taking in the present running performances for such proton accelerators. Figure 4 shows the expected distribution of the energy of $v_{e}^{C C}$ for $1.0 \times 10^{20}$ pot and a $500 \mathrm{t}$ detector placed $100 \mathrm{~m}$ downstream of the tagger most upstream point. The distributions of the oscillated $v_{e}^{C C}$ for Hyper-K and DUNE are also shown in black dotted and dashed curves. The different distributions illustrate how shifting the detector off-axis with respect to the Kaon beam could be used to get a better sampling the lower energy region. The possibility of a broad and flexible energy coverage is also being studied within Work Package 1 by acting on the kinematics of the hadronic beam (Sect. 4).

Event rates. $0.5 \mathrm{kt}, 1.0 \mathrm{e}+20 \mathrm{pot}, \mathrm{L}=0.1 \mathrm{~km}$

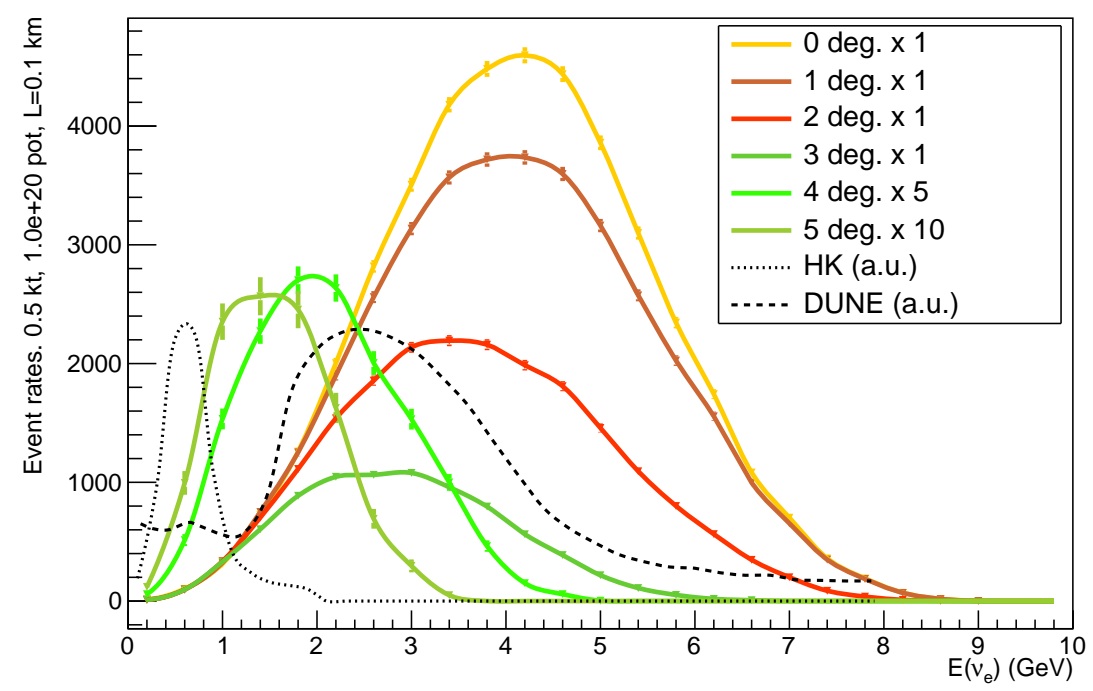

Figure 4: $v_{e}^{C C}$ events at the neutrino detector (500 tons) as a function of the neutrino energy. The beamline is optimized for the energy range of interest for DUNE. The rates are shown at different off-axis angles of the detector $\left(0^{\circ}-5^{\circ}\right)$. The events correspond to $1 \times 10^{20}$ pot at the Fermilab Main Ring $(120 \mathrm{GeV})$ and the detector is located $50 \mathrm{~m}$ after the beam dump $(0.1 \mathrm{~km}$ after the entrance of the decay tunnel). The dotted and dashed lines show the Hyper-Kamiokande and DUNE neutrino spectrum, respectively.

\section{Development and tests of the positron tagger prototypes}

The basic calorimetric unit (Ultra-Compact Module - UCM in Fig. 5, top.) is made of five, 15$\mathrm{mm}$ thick, iron layers interleaved by 5 -mm thick plastic scintillator tiles. The transverse dimension is $3 \times 3 \mathrm{~cm}^{2}$ for a $10 \mathrm{~cm}$ length $\left(4.3 X_{0}\right)$. Nine wavelength shifting (WLS) fibers crossing the UCM are connected directly to $1 \mathrm{~mm}^{2} \mathrm{SiPM}$ (Fig. 5, bottom left). The fibers are inserted into a 3D printed plastic mask located downstream of each UCM. Suitable grooves in the mask are used to host the SiPMs that are soldered on a printed circuit board and protected with an epoxy layer (Fig. 5, bottom right). The coupling is done in air. Unlike conventional shashlik calorimeters this scheme avoids the occurrence of large passive regions usually needed to bundle the fibers and route them to a common photo-sensor. The output signals of the SiPMs are not amplified and simply summed and brought to the front-end electronics (fast digitizers). This readout offers outstanding flexibility in terms of choice of granularity and homegeneity in the longitudinal shower sampling. 


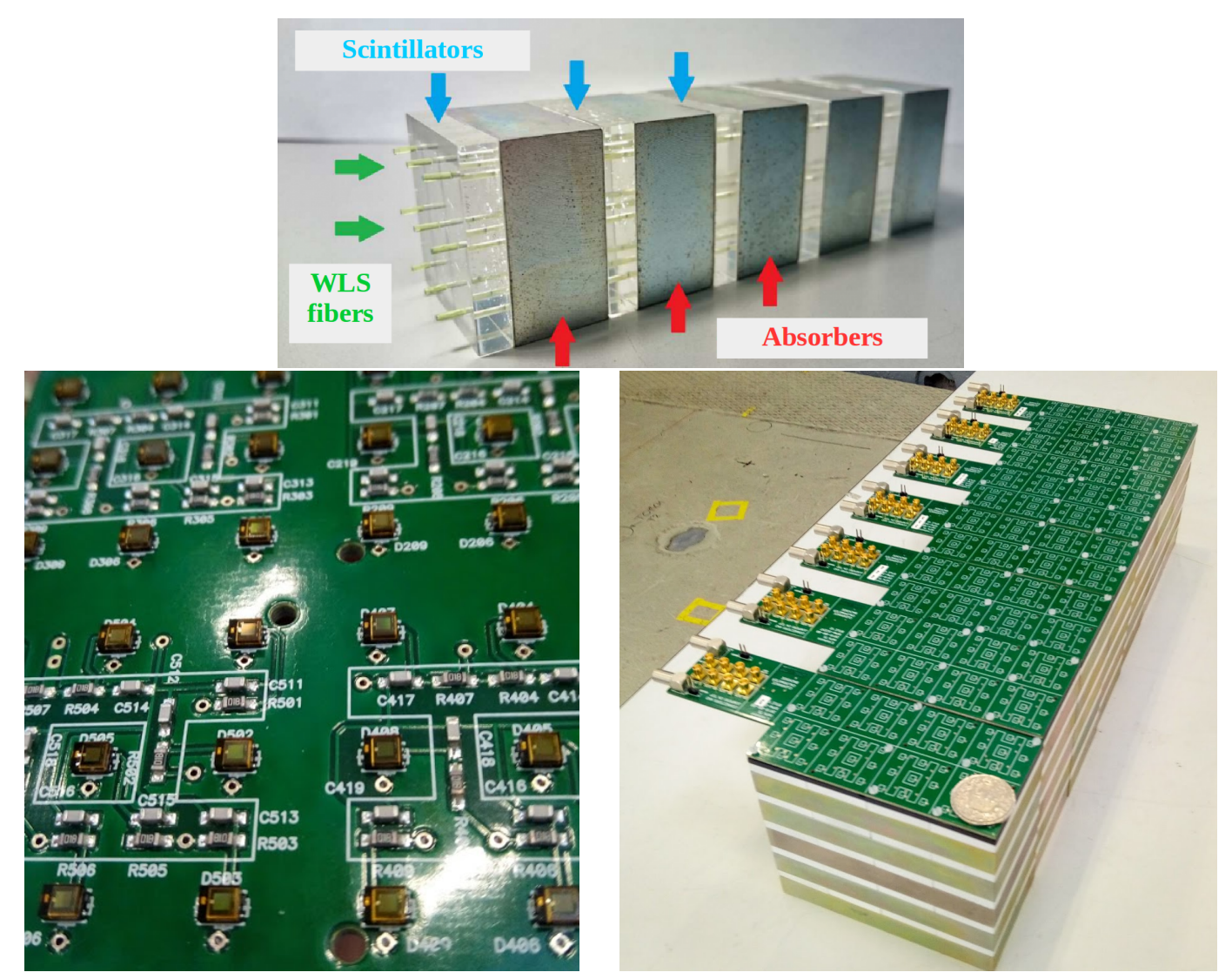

Figure 5: (Top): a single UCM with $15 \mathrm{~mm}$ thick iron absorbers, $5 \mathrm{~mm}$ thick plastic scintillators $(3 \times 3 \times$ $10 \mathrm{~cm}^{3}$ ) and 9 WLS fibers with a spacing of $1 \mathrm{~cm}$. (Bottom Left): view of a printed circuit board (PCB) with FBK $20 \mu \mathrm{m}$ cell $1 \times 1 \mathrm{~mm}^{2}$ SiPMs mounted in a matrix pattern with a $1 \mathrm{~cm}$ spacing. (Bottom Right): Seven modules composed of eight UCMs each with PCB boards hosting SiPMs already mounted (rotated by $90^{\circ}$ with respect to the exposure configuration of Fig. 7).

\section{SiPM neutron irradiation at INFN-LNL}

In our reference option for the tagger instrumentation SiPMs are foreseen to be embedded in the bulk of the calorimeters and, hence, will be exposed to radiation. Since these devices are in particular sensitive to neutron damage, we performed irradiation tests at the CN facility at INFNLNL (Legnaro) in June 2017. Large neutron fluences (up to $10^{12} \mathrm{n} / \mathrm{cm}^{2}$ in less than one day) were achieved by impinging $5 \mathrm{MeV}$ protons accelerated by the $\mathrm{CN}$ Van de Graaff generator to a thin Beryllium target. The target area with concrete shielding is shown in Fig. 6, left. SiPMs of different pixel sizes $(12,15,20 \mu \mathrm{m})$ produced by the Fondazione Bruno Kesserl (FBK) were exposed and characterized in terms of $\mathrm{i}-\mathrm{V}$ curves at increasing irradiation (Fig. 6, right). Noise waveforms were also sampled before and at intermediate irradiation levels. These SiPMs were later mounted on UCM modules and the response to mips and electrons tested at the CERN East Area facility in August and October 2017 [14]. 

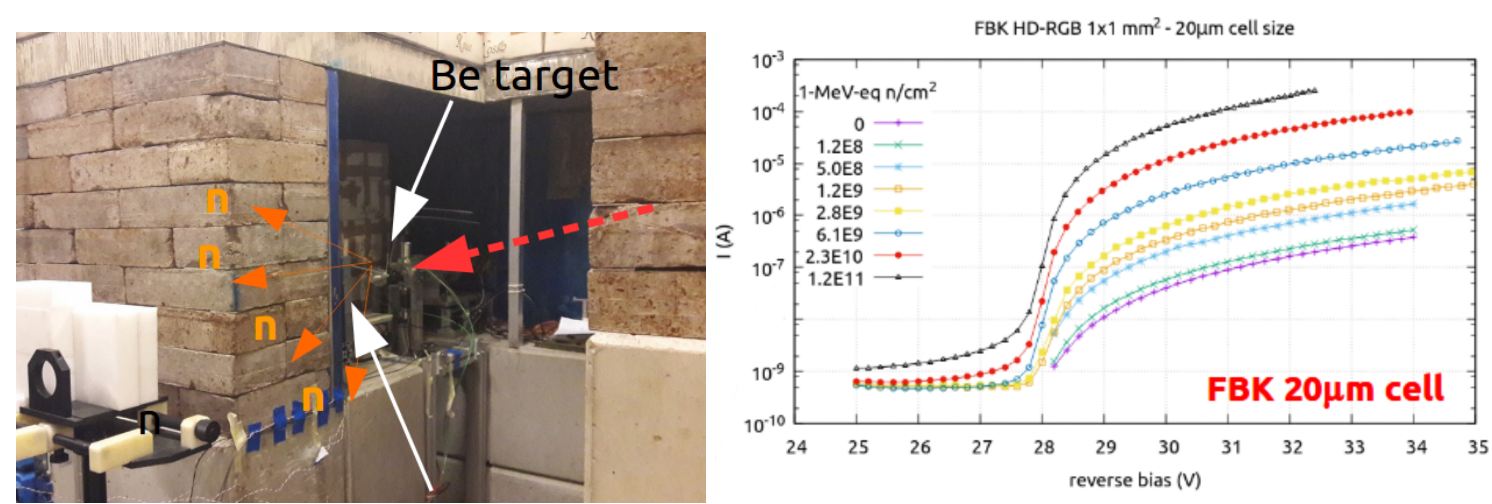

Figure 6: (Left): A picture of the target region at INFN-LNL used to produce large neutron fluxes for SiPM irradiation by impinging $5 \mathrm{MeV}$ protons from the $\mathrm{CN}$ accelerator on Beryllium. (Right): Dependence of the measured current (A) on the reverse bias voltage for a $20 \mu \mathrm{m}$ cell SiPM from FBK for various values of the estimated neutron irradiation in $1 \mathrm{MeV}-\mathrm{eq} / \mathrm{cm}^{2}$ neutrons).

\section{Tests with particle beams at the CERN East area}

UCM prototypes have been characterized with cosmic rays and with charged particles $(e / \pi / \mu)$ in the 1-5 GeV range at the CERN-PS East Area facility (T9 beamline) in several exposures in 2016 (July-November) and 2017 (August-October) [11, 12, 13].

The test beam instrumentation includes: a pair of Silicon tracking chambers allowing to select single particles and determine their impact point in the calorimeter for fiducialization [9]; two Cherenkov threshold chambers to tag electrons and muons and a pair of scintillators put downstream of the calorimeter, to further tag passing-through muons. The readout has been performed with commercial digitizers by CAEN with a sampling granularity ranging fron 0.5 to $4 \mathrm{~ns}$. The collaboration is working to develop prototypes for custom electronics to perform triggerless digitization of UCM waveforms over the proton spill duration.

The measurements at test beams are intended to characterize the capabilities of several setups in terms of energy resolution and linearity of response for electromagnetic showers, the uniformity of modules and the performaces of the self-calibration with minimum ionizing particles. Most importantly, the capability of the GEANT4 simulation to reproduce the data distributions is tested in depth. Several technological solutions have been tested: the choice of the scintillator materials (EJ200, EJ204, Uniplast) and thicknesses (5-10 mm) of the WLS fibers (Kuraray Y11-MC, St. Gobain BCF-92-MC) as well as the solutions for an optimal coupling of the fibers with the SiPM sensors and the choice of optical elements (reflectors for WLS fiber ends, TiO or Tyvek diffusers around scintillators). Injection molded scintillators that are suitable for large scale production have been successfully produced by Uniplast (Russia) and tested at CERN in July 2017. In 2017 new prototypes have been tested by employing the SiPMs irradiated at INFN-LNL (see Sect. 7) to assess the recovery time and radiation hardness requirements.

During the November 2016 CERN test beam a module composed of 56 UCMs in 7 longitudinal layers $\left(\sim 30 X_{0}\right.$, Figs. 5, 7) and of an outer module acting as an energy catcher, were exposed to $e^{-} / \pi^{-}$with grazing incidence at various incidence angles from 0 to $200 \mathrm{mrad}$ (Fig. 7, left). The 
distribution of the tracks impact parameters on the front face of the calorimeter as determined by the silicon chambers is shown in Fig. 7, right. The electron/pion separation capabilities in data and Monte Carlo have been determined at various incident angles [10]. In Fig. 9 (left) the distribution of the total visible energy for a $100 \mathrm{mrad}$ tilt of the calorimeter and a $4 \mathrm{GeV}$ beam of negative pions, muons and electrons is shown for data (bullets) and Monte Carlo (histograms, broken down by particles). Fig. 9 (right) shows the energy resolution for electrons in data and Monte Carlo. The baseline option for the $t_{0}$-layer (plastic scintillator doublets) has also been tested with several layouts for light collection (direct SiPM, one or two WLS in several geometries and thicknesses).

The results of the test $[10,13,14]$ demonstrate that the design is appropriate for the needs of positron monitoring and validate the full simulation of the decay tunnel. A full-scale demonstrator is foreseen to be built by the end of the project, with a length of $\sim 3 \mathrm{~m}$ and an azimuthal coverage of $180^{\circ}$, including the photon veto (Fig. 1, right) for the identification of $\gamma$ originating from $\pi^{0}$.
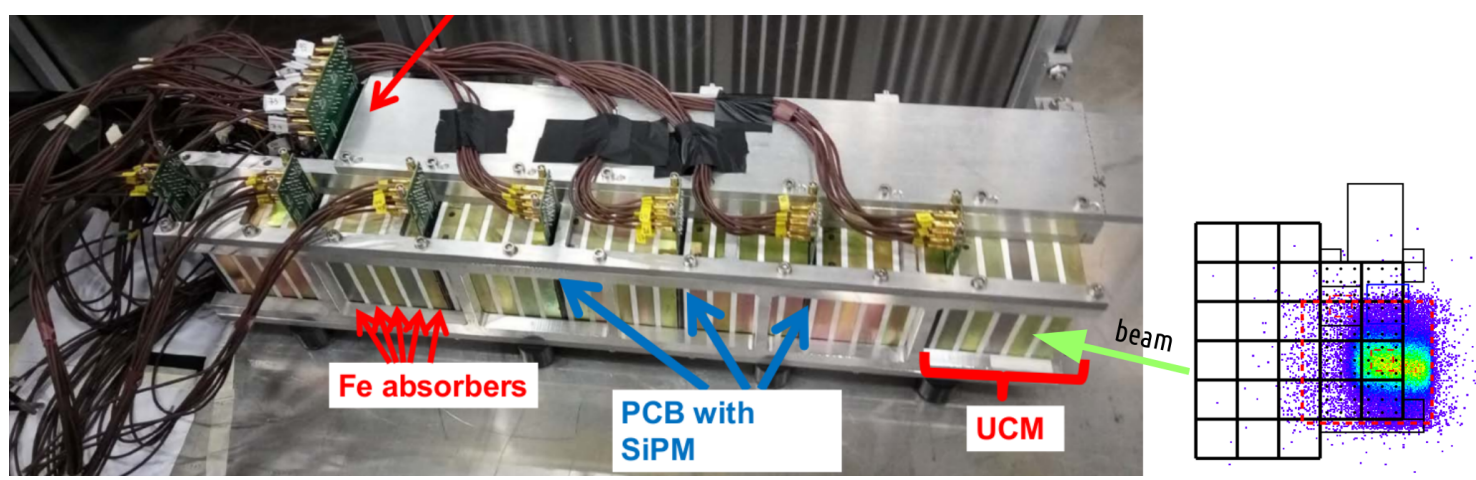

Figure 7: (Left): The prototype exposed at the CERN-PS T9 particle beams. The calorimeter is composed of an inner part (bottom in the picture) comprising seven calorimetric blocks (those of Fig. 7, bottom right) and a $60 \mathrm{~cm}$ long "hadronic" block for the outer part (up in the picture). The compact layout of the light readout is visible through the cabled PCBs. The system was mounted on a tunable mechanical cradle to sample easily various impinging angles for the particle beam. (Right): Distribution of the tracks impact parameters on the front side of the calorimeter as reconstructed by the Silicon tracking chambers using the $5 \mathrm{GeV}$ beam. The layout of the calorimeter is superimposed: each square represents a $3 \times 3 \mathrm{~cm}^{2} \mathrm{UCM}$.

Non conventional options based on polysiloxane scintillators [16] to avoid drilling or injection molding and allowing an improved radiation hardness are also being scrutinized. A prototype polysiloxane shashlik calorimeter composed of three modules $\left(6 \times 6 \times 15 \mathrm{~cm}^{3}\right.$ each, $13 X_{0}$ in total $)$ consisting of $12 \mathrm{UCM}$ units and a $15 \mathrm{~mm}$ sintillator thickness was built at INFN-LNL and exposed to particle beams at the CERN East area (Fig. 8, left). The absolute light yield and uniformity are very promising (Fig. 8 , right).

\section{Conclusions}

The final goal of ENUBET (2016-2021) is to demonstrate that $e^{+}$-monitored $v_{e}$ beams can be built using existing technologies and hosted at CERN, Fermilab or J-PARC for a new generation of experiments on neutrino cross sections. The results obtained in the preparatory and initial phase of the project are very encouraging. The design and simulation of the hadronic beamline has started and the preliminary results support or improve the initial estimates of the achievable event 

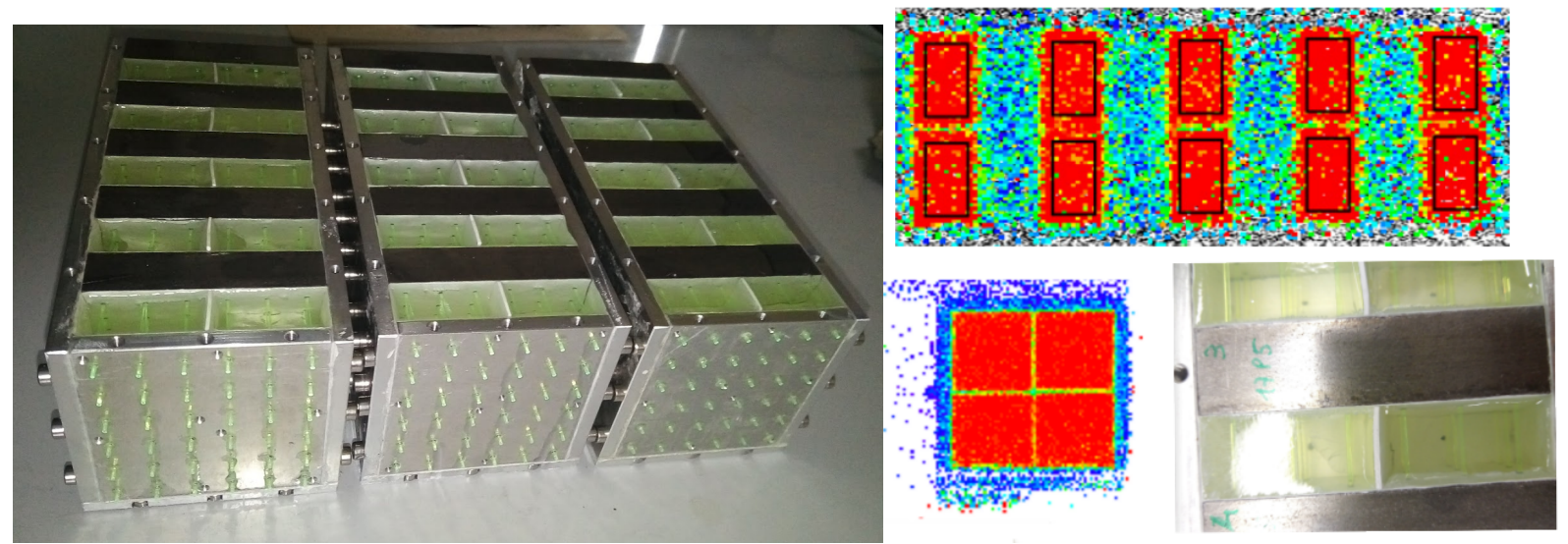

Figure 8: (Left): Polysiloxane-based shashlik calorimeter in three modules $\left(6 \times 6 \times 15 \mathrm{~cm}^{3}\right.$ each) consisting of 12 UCM units for a total thickness of about $13 X_{0}$. (Right): Detail of two layers and bi-dimensional efficiency maps (frontal and lateral) obtained with mip tracks at the CERN-PS-T9 test beam (Oct. 2017).
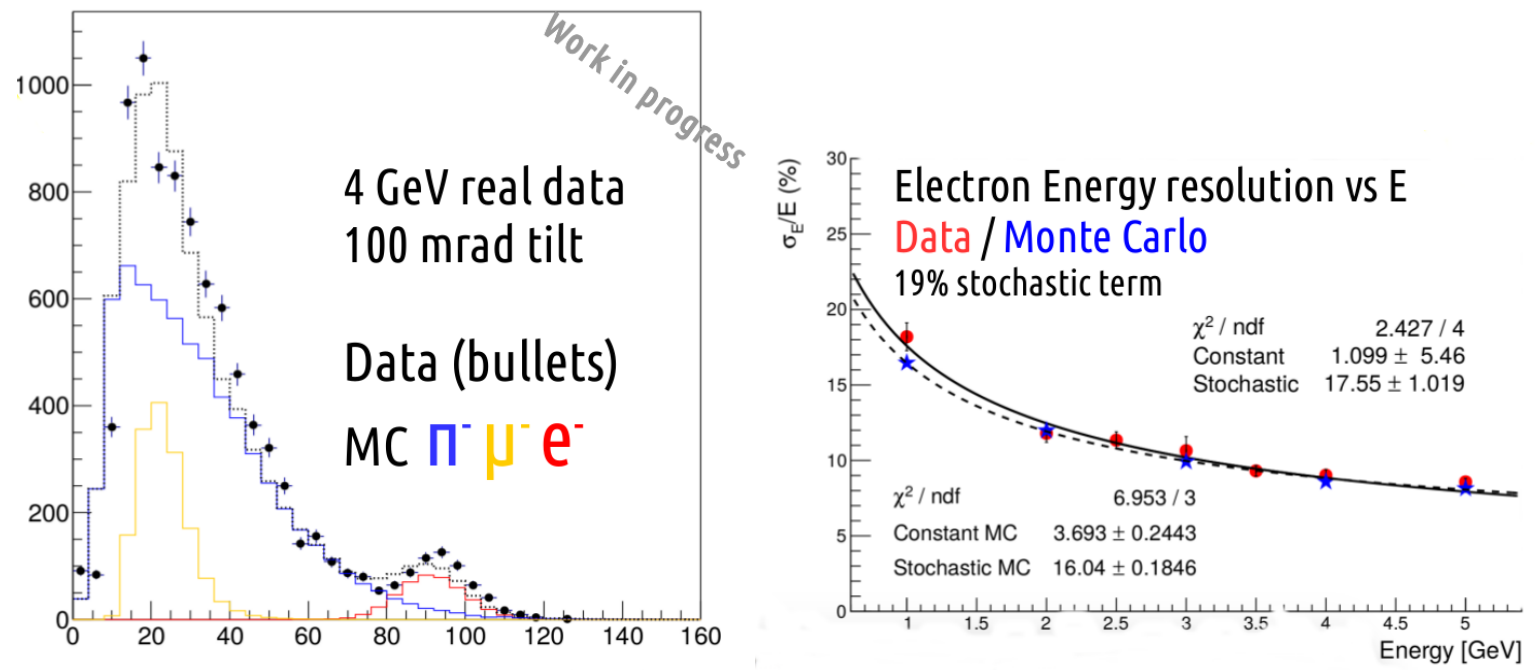

Figure 9: (Left): Data and Monte Carlo distributions of the total visible energy for a $100 \mathrm{mrad}$ tilt of the calorimeter and a $4 \mathrm{GeV}$ beam of negative pions, muons and electrons in the CERN-PS T9 beamline. (Right): Energy resolution for electrons in data (red point) and Monte Carlo (blue). The fitted parametrization follows the standard form: $\frac{\sigma(E)}{E}=\frac{s}{\sqrt{E}} \oplus c$.

rates. Full simulations support the viability and effectiveness of the calorimetric approach whereas prototypes tests demonstrate that shashlik calorimeters with longitudinal segmentation can fulfill the requests for $e^{+}$monitoring in the relevant energy region of few $\mathrm{GeV}$.

\section{Acknowledgments}

This project has received funding from the European Union's Horizon 2020 Research and Innovation programme under Grant Agreements no. 681647 and 654168. 


\section{References}

[1] A. Longhin, L. Ludovici and F. Terranova, A novel technique for the measurement of the electron neutrino cross section, Eur. Phys. J. C 75, 155 (2015).

[2] A. Berra et al. [ENUBET Coll.], Enabling precise measurements of flux in accelerator neutrino beams: the ENUBET project, CERN-SPSC-2016-036; SPSC-EOI-014.

[3] K.L.Brown et al., TRANSPORT, A computer program for designing charged particle beam transport systems, May 1983, http://lss.fnal.gov/archive/nal/fermilab-nal-091.pdf.

[4] The FLUKA Code: Developments and Challenges for High Energy and Medical Applications, T.T. Böhlen, F. Cerutti, M.P.W. Chin, A. Fassó, A. Ferrari, P.G. Ortega, A. Mairani, P.R. Sala, G. Smirnov and V. Vlachoudis, Nuclear Data Sheets 120, 211-214 (2014).

[5] FLUKA: a multi-particle transport code, A. Ferrari, P.R. Sala, A. Fassó, and J. Ranft, CERN-2005-10 (2005), INFN/TC_05/11, SLAC-R-773.

[6] S. Agostinelli et al., GEANT4: a simulation toolkit, Nucl. Instrum. Meth. A 506, 250 (2003).

[7] A. Meregaglia et al., ENUBET: Enhanced NeUtrino BEams from kaon Tagging, JINST 11 (2016) C12040.

[8] F. Pupilli et al., Positron identification in the ENUBET instrumented decay tunnel, NEUTEL 2017, these proceedings.

[9] M. Prest et al., The AGILE silicon tracker; an innovative $\gamma$-ray instrument for space, Nucl. Instr. and Meth. in Phys. Res. A, 501, 280-287 (2003).

[10] A. Berra et al., Testbeam performance of a shashlik calorimeter with fine-grained longitudinal segmentation, in preparation.

[11] A. Berra et al., A compact light readout system for longitudinally segmented shashlik calorimeters, Nucl. Instrum. Methods A 830, 345 (2016).

[12] A. Berra et al., Longitudinally segmented shashlik calorimeters with SiPM readout, Nucl. Instrum. Methods A 845, 511 (2017).

[13] A. Berra et al., Shashlik Calorimeters With Embedded SiPMs for Longitudinal Segmentation, IEEE Trans. Nucl. Sci. 64, 1056 (2017).

[14] V. Mascagna, Talk at the $8^{\text {th }}$ International Conference on New Developments in Photodetectors (NDIP 2017), Tours, France, 3-7 July 2017.

[15] T. J. Roberts et al., G4Beamline: particle tracking in matter-dominated beamlines. Proceedings of EPAC08, Genoa, Italy. http://g4beamline.muonsinc.com.

[16] S. Carturan et al., Novel polysiloxane-based scintillators for neutron detection, Radiation protection dosimetry, 2010, 11, 471-6 143. 